\title{
Time Stability and Coherence Analysis of Multiunit, Single-Unit and Local Field Potential Neuronal Signals in Chronically Implanted Brain Electrodes
}

\author{
Gaurav Sharma, ${ }^{1}$ Nicholas Annetta, ${ }^{1}$ David Friedenberg, ${ }^{1}$ Tony Blanco, ${ }^{1}$ Daphne Vasconcelos, ${ }^{1}$ \\ Ammar Shaikhouni, ${ }^{2}$ Ali R Rezai, ${ }^{2}$ and Chad Bouton ${ }^{1^{*}}$ \\ ${ }^{1}$ Battelle Memorial Institute, Columbus, Ohio, United States of America; ${ }^{2}$ Department of Neurosurgery and the Center for \\ Neuromodulation at The Ohio State University, Columbus, Ohio, United States of America; and *current affiliation: The Feinstein \\ Institute for Medical Research, Manhasset, New York, United States of America
}

\begin{abstract}
Introducing neural sensing and decoding to open-loop neurostimulation technologies has the potential to significantly improve the diagnosis and treatment of a wide variety of diseases treated through bioelectronic medicine. Chronically implanted multielectrode arrays (MEA) can be used for such neural sensing and are critical for obtaining data of high spatial and temporal resolution to provide accurate decoding. Signals recorded from these arrays include local field potentials (LFP), and multiunit (MU) and single-unit (SU) activity. LFP offer signal stability over time, but at the expense of decreased spatial resolution. SU activity, on the other hand, offers better spatial resolution, but is considered less stable in chronic applications. MU activity, which represents an aggregate spiking activity of a population of neurons on the order of several hundred microns away from the recording tip, is considered a signal that can offer a compromise between the two signals. Here we used a wavelet decomposition method to extract and characterize the LFP, MU and SU signals obtained from a 96-channel MEA implanted in the motor cortex of a nonhuman primate over a 7.5-month period. We observed that not only are the MU signals more stable over time compared with SU activity, but that they are also significantly less correlated among electrodes compared with LFP over the spatial scale of the implanted array. Histological analysis of tissue sections also revealed a 51\% reduction in the number of neuronal cell bodies within a $100-\mu \mathrm{m}$ radius around the electrode tips of the implanted tissue compared with control tissue. Our results indicate that MU activity offers long-term signal stability with less correlated signals, potentially providing an effective signal for neural sensing in bioelectronic medicine.
\end{abstract}

Online address: www.bioelecmed.org

doi: 10.15424/bioelectronmed.2015.00010

\section{INTRODUCTION}

Bioelectronic medicine is a rapidly growing discipline where the aim is to develop technologies and devices for the diagnosis and treatment of diseases through neurostimulation and neurosensing in the central and peripheral nervous system. There is increasing evi- dence that introducing neural sensing and decoding to open-loop neurostimulation technologies can improve the diagnosis and treatment of a wide variety of diseases treated by bioelectronic medicine by enabling a closed-loop control of the spatial and temporal characteristics of the stimulation (1). Chronically im-

Address correspondence to Gaurav Sharma, Battelle Memorial Institute, 505 King

Avenue, Columbus, OH 43201. Phone: 614-424-3259; Fax: 614-458-3259; E-mail: sharmag@battelle.org, or Chad Bouton, The Feinstein Institute for Medical Research, 350 Community Drive, Manhasset, NY 11030. Phone: 614-561-1140; Fax: 516-562-1022; E-mail: cbouton@nshs.edu. Submitted August 24, 2015; Accepted for publication September 8, 2015; Published Online (www.bioelecmed.org) September 9, 2015.

\section{The Feinstein Institute for Medical Research Empowering Imagination. Pioneering Discovery.}

planted neural interfaces may be used for such neural sensing and are critical for obtaining data of high spatial and temporal resolution; therefore, it is crucial to understand their signal recording characteristics over time. Current neural interfaces such as multielectrode arrays (MEA) can utilize a number of neural signals for decoding and control. These signals include local field potential (LFP), multiunit (MU) and single-unit (SU) activity $(2,3)$. SU activity, while providing excellent spatial and temporal resolution, is susceptible to degradation over time (4-6). Inflammatory tissue responses, which result in the formation of an insulative glial sheath around the recording electrodes, and subsequent local neurodegeneration, have been suggested as the primary reasons for SU signal degra- 
dation (7-10). By contrast, LFP activity, which represents a summation of excitatory and inhibitory dendritic potentials of hundreds or thousands of neuronal processes in a "listening sphere" around the tip of the electrode $(11,12)$, can be easier to record over long periods of time and can thus provide a reliable signal for neuroprosthetics $(12,13)$. However, LFP suffers from poor spatial resolution as multiple electrodes recording from the same neurons can pick up highly correlated signals (14).

Multiunit activity is the third type of signal that can be obtained from an implanted MEA. MU represents an aggregate spiking activity of a population of neurons on the order of several hundred microns away from the electrode tip and can be estimated without explicit spike detection (14). Compared to SU, analyzing MU activity not only can provide a more robust signal for chronic central/peripheral nervous system implants, but also can be a more efficient way to study neural dynamics and can better represent the interaction between neuronal processes $(14,15)$. Other groups have developed methods to analyze MU activity. Choi et al. defined MU activity as the bandpass-filtered signal (300 to $3000 \mathrm{~Hz}$ ) and used discrete wavelet transformation to analyze MU activity without spike detection from a rodent cortex (15). Stark et al. defined and obtained MU signals by computing the root mean square (RMS) in the frequency band of 300 to $6000 \mathrm{~Hz}$ from an electrode implanted in the premotor cortex of a nonhuman primate and used it to predict arm movements (14). However, to the best of our knowledge, there are no studies on the systematic characterization of MU signals, a comprehensive analysis of signal quality, stability and coherence of SU, MU and LFP activity, and their correlation with tissue histology around implant sites.

In this study, we used the wavelet decomposition method to extract and characterize the SU, MU and LFP signals obtained from a 96-channel MEA implanted in the motor cortex of a nonhuman pri- mate over a 7.5-month period. Wavelet decomposition is a technique for decomposing a signal that provides both frequency and temporal information, and has been shown to be an effective way to process neural signals (15-18). Wavelets are also efficient because they provide information across a large range of frequency bands, encompassing SU, MU and LFP, without requiring spike sorting. We compared the spectral and temporal characteristics of MU signal with those of SU and LFP activity. We also investigated the correlation of electrophysiological signals with tissue impedance and examined neuronal density around electrodes after chronic implantation. We observed a steady decline in the total number of SUs being recorded over the 7.5-month period that correlated with the reduction in number of neuronal cell bodies observed in tissue sections in postmortem histology. More importantly, we observed that the MU signal stability over time was either comparable to or better than SU signal stability over time. In addition we found the MU signals to be significantly less correlated compared with LFP, indicating a higher unique information content of MU signals. Our results indicate that MU signals offer a balance between long-term signal stability and unique information content, and could provide an effective signal for long-term neural sensing and decoding in bioelectronic medicine.

\section{MATERIALS AND METHODS}

\section{Microelectrode Array and Surgical Implantation}

A 96-channel microelectrode array (Blackrock Microsystems) was used in this study. This array has a $4.4-\mathrm{mm} \times$ 4.2-mm platform and has 100 electrodes, out of which 96 are available for recording. The electrodes are $1.5 \mathrm{~mm}$ in length, spaced $400 \mu \mathrm{m}$ apart and are coated with Parylene-C as an insulator with $\sim 40 \mu \mathrm{m}$ exposed Pt-IrOx tips. One male Cynomolgus monkey (weight $\sim 5.5 \mathrm{~kg}$ ) was used for this study. All surgical procedures were performed using standard sterile procedures in an approved animal facility with a dedicated surgery suite. The animal was maintained in an AAALAC (Association for Assessment and Accreditation of Laboratory Animal Care) accredited facility. All procedures were approved by the Battelle's IACUC (Institutional Animal Care and Use Committee). The array was implanted in the M1 motor cortex using standard neurosurgical techniques as described previously (19). Briefly, a wide enough craniotomy was performed to expose the arcuate sulcus and to allow implantation of the array in the precentral gyrus. The dura matter was exposed and dissected to expose the brain cortex. The array was positioned above the cortex and was inserted rapidly into the cortex using a pneumatic insertion device. Figure $1 \mathrm{~A}$ is a schematic showing the approximate location of the implanted electrode with respect to the M1 motor cortex and the central sulcus. Figure 1B shows the actual image of the electrode setup and craniotomy. The array and the cortex were covered with the dura matter and sutured. Small pieces of absorbable hemostatic gelatin sponge and the piece of bone flap from the craniotomy were then fixed in place with titanium straps and screws. Bone cement was applied to seal the edges and any defects. Finally, the microarray wires and connector were secured to the skull with screws. The animal was allowed a 2-wk recovery period after the surgery, during which it was allowed free access to food and water.

\section{Recording and Signal Processing}

Recording sessions which included neural recordings and impedance measurements were started $24 \mathrm{~d}$ after the implant to allow for postsurgical recovery. Data were recorded approximately every 2 wks for the duration of the experiment. No data were recorded during wks 7 or 8 . Neural data were acquired and partially processed using the NeuroPort System (Blackrock Microsystems), consisting of a front-end amplifier/digitizer and a neural signal processor. The front-end amplifier uses a first order $0.3-\mathrm{Hz}$ high 
A

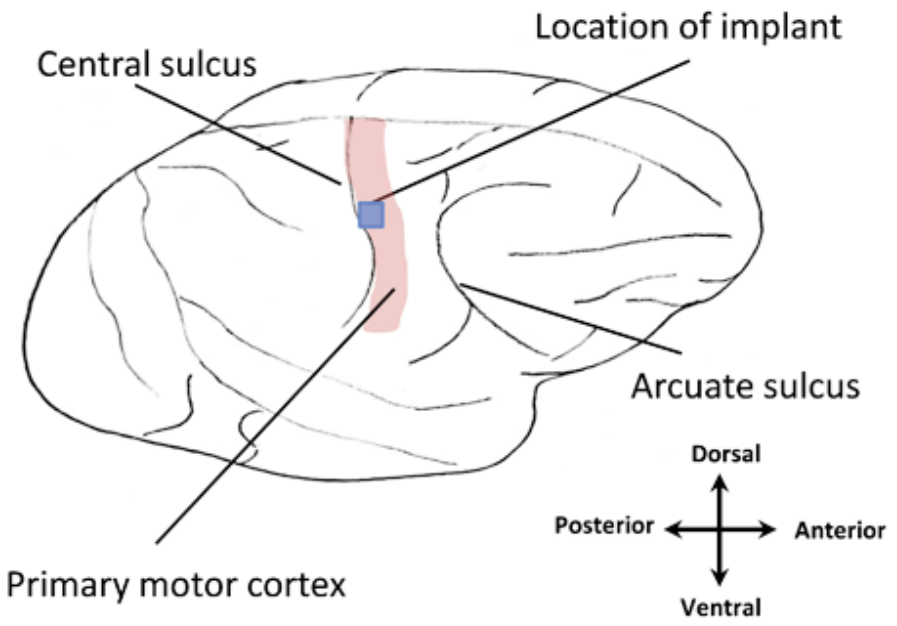

B

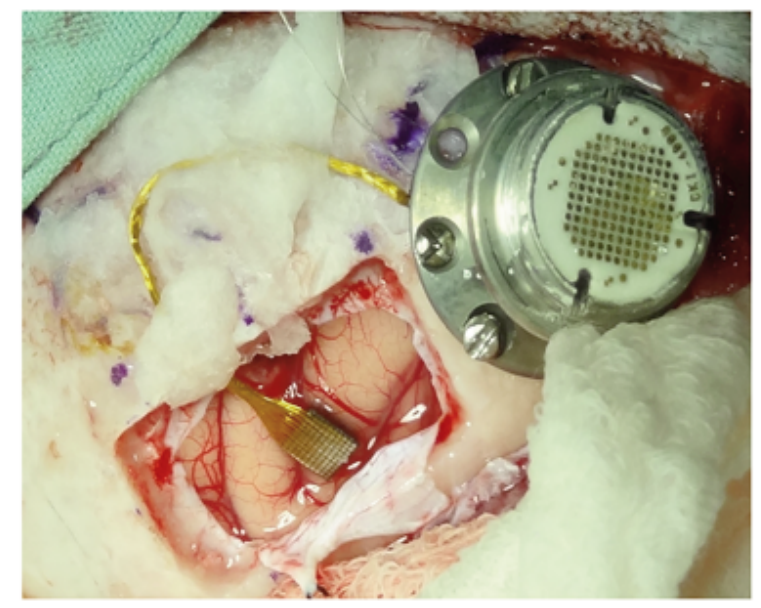

Figure 1. Multielectrode array (MEA) implantation. A 96-channel MEA (1.5 mm probe length, $400 \mu \mathrm{m}$ spacing) is implanted in the primary motor cortex of a monkey. (A) Approximate location of the implanted array showing the landmarks used to locate the implantation site in the primary motor cortex; (B) Craniotomy and array after insertion.

pass filter along with a third order Butterworth $7.5 \mathrm{kHz}$ low pass filter. The digitizer has 16-bit resolution at $0.25 \mu \mathrm{V} /$ bit. Real-time neural signal processing was performed using the Central software suite (Blackrock Microsystems) and post processing was performed using custom code in MATLAB (The Mathworks). Neural recordings were performed throughout the study. No cues were given to the animal during recording. On the day of recording, electrode impedances were measured and continuous data were collected. Electrode imped- ances were measured at $1 \mathrm{kHz}$ using Blackrock's Impedance Tester tool. Before recording continuous data, each electrode channel was manually searched for neural spikes. Spikes were sorted using the manual hooping method provided by the Blackrock software. The same operator sorted spikes during all recording sessions. Continuous data were recorded for $90 \mathrm{~s}$ at a sampling rate of 30,000 samples/second. The continuous data were filtered in software using a fourth order Butterworth $250 \mathrm{~Hz}$ High Pass filter before extracting 1.6-ms windows around each spike to collect individual spike waveforms. The signal-tonoise ratio (SNR) was calculated as the ratio between the peak-to-peak voltage amplitude of the mean spike waveform and the standard deviation of the background noise.

\section{Wavelet Analysis}

MATLAB was used to calculate a 10level wavelet representation of the continuous data using the Daubechies wavelet of length four (Db4) (20). The 10 levels provide a good representation of the frequency space, which will be discussed further in the Results section. There are many possible choices of wavelets and different wavelets will be optimal for different situations. The $\mathrm{Db} 4$ wavelet was selected because it is commonly used and easy to compute $(21,22)$. For the purpose of comparison, a unitybased normalization was also performed on the mean wavelet power to scale the data between 0 and 1 . We classified the signal into SU (>1 kHz), MU (300 Hz to $1 \mathrm{kHz}$ ) and LFP (1 to $200 \mathrm{~Hz}$ ) based on previous classification $(14,15)$ and our approximate wavelet frequency subbands as shown in Table 1.

\section{Coherence Analysis}

Coherence analysis was performed to estimate the degree of signal independence in the neural signals recorded by electrodes in the SU, MU and LFP frequency domains. A coherence value of

Table 1. Wavelet levels and the corresponding frequency subbands.

\begin{tabular}{lc}
\hline Wavelet level & Frequency subband \\
\hline$W_{0}$ & $7.5 \mathrm{k}-15 \mathrm{kHz}$ \\
$W_{1}$ & $3.75 \mathrm{k}-7.5 \mathrm{kHz}$ \\
$W_{2}$ & $1.87 \mathrm{k}-3.75 \mathrm{kHz}$ \\
$W_{3}$ & $938-1.87 \mathrm{kHz}$ \\
$W_{4}$ & $469-938 \mathrm{~Hz}$ \\
$W_{5}$ & $235-469 \mathrm{~Hz}$ \\
$W_{6}$ & $117-235 \mathrm{~Hz}$ \\
$W_{7}$ & $58.6-117 \mathrm{~Hz}$ \\
$W_{8}$ & $29.3-58.6 \mathrm{~Hz}$ \\
$W_{9}$ & $0-29.3 \mathrm{~Hz}$ \\
\hline
\end{tabular}


zero signifies that the two signals are completely uncorrelated (or independent) while unit coherence signifies that the two signals are in perfect correlation. Coherence was calculated using a custom script and using the mscoherence function in MATLAB. Coherence was estimated between all possible pairs of electrodes in the three frequency domains and plotted as a function of the interelectrode distance.

\section{Histology}

The anesthetized animal was euthanized with sodium pentobarbital. The implant site was uncovered surgically using an oscillating saw, and the implant was removed within an approximately $2-\mathrm{cm}^{2}$ block of brain (cerebral cortical) parenchyma that was $8 \mathrm{~mm}$ in depth. The implant and cerebral parenchyma were fixed as one block in $10 \%$ neutralbuffered formalin, trimmed to approximately $1 \mathrm{~cm}^{2}$, and processed into a paraffin block. Approximately $5 \mu \mathrm{m}$ thick sections were cut starting at the electrode tips (that is, in the deep cortex). Sectioning continued through the electrode tips and shafts, with each successive section being more superficial within the cortex than the last. Serial sections were kept and stained with hematoxylin and eosin (H\&E) stain for microscopic examination. Microscopic measurements were made between electrode tips to determine the degree of shrinkage due to fixation (shrinkage factor), and this factor was used to determine actual distances between electrode tips and individual neurons. Using an ocular micrometer, the distance was measured from the first appearance of each electrode tip in the brain tissue (that is, the tip of the electrode) to each identifiable neuron within $100 \mu \mathrm{m}$ from the center of the tip. Only electrode tips that appeared to be within the pyramidal neuron layer (Layer V), and did not have extensive artifact (tearing of section, folds, and so on), were used for counts and measurements. Only neurons with nuclear profiles in the plane of section were counted and used for measurements. On each slide, the number of neurons within a $100-\mu \mathrm{m}$ radius of two random points within the cerebral cortex adjacent to the electrode array, both taken at a level similar to that of the electrode tips (based upon the presence of pyramidal neurons) were used for control measurements. Neurons were identified based upon morphology: size, presence of Nissl substance and nuclei that had a prominent nucleolus. Neurons counted included those of nonpyramidal morphology. Data were entered into a spreadsheet for tabulation and statistical analyses.

\section{Statistical Analysis}

Data is presented using mean and standard error of the mean (mean \pm SEM). For all tests, a two-tailed criterion was used and $p<0.05$ was considered significant.

All supplementary materials are available online at www.bioelecmed.org.

\section{RESULTS}

\section{Electrophysiology Recordings}

Figure 2A shows an example of multiple neuronal action potential (spikes) recorded from the same electrode. The spikes are overlaid on one another and sorted as different units are plotted in different colors. These spikes are the result of random firing of neurons (single units) during the recording session and indicate the viability of the electrode (5). We also estimated the total number of SUs being recorded over the course of the experiment. A total of 53 SUs were recorded from the array at $24 \mathrm{~d}$ postimplantation. A steady decline in SU count was observed over time, leading to only 6 SUs being recorded at $227 \mathrm{~d}$ postimplantation (Figure 2B). In addition to SU count, we also calculated the firing rate of neurons. Firing rate of a given unit was calculated as the total number of spikes divided by the total recording time. After an initial increase, a peak mean firing rate of $19.9 \pm$ 2.5 spikes/second was estimated $90 \mathrm{~d}$ postimplantation, which then declined to $7.1 \pm 1.33$ spikes/second at $227 \mathrm{~d}$ postim- plantation (Figure 2C). As a quantitative measure of the reliability of the neural signals, we also analyzed the signal-tonoise ratio (SNR) of spikes over the course of the study. SNR fluctuated over recording sessions but overall we observed a significant trend (slope $=-0.01$, $R^{2}=0.78, p<0.001$ ) of declining SNR over time from a peak value of $4.97 \pm 0.54$ at $\mathrm{d} 31$ to $2.61 \pm 0.54$ at $\mathrm{d} 227$ (Figure 2D).

The mean impedance value of the electrodes at $24 \mathrm{~d}$ postimplantation was $1.59 \pm 0.47 \mathrm{M} \Omega$ which dropped sharply to $282 \pm 1.34 \mathrm{k} \Omega$ by $73 \mathrm{~d}$ post implantation and stabilized thereafter to an average value of $273 \mathrm{k} \Omega$ for the remaining duration of the study (See Supplementary Figure S1).

\section{Histology}

The animal was euthanized at the end of the experiment and a $2-\mathrm{cm}$ square block of cortical tissue containing the MEA was explanted and examined. The dissected tissue block was sectioned, stained and analyzed under a microscope to determine the number of neuronal cell bodies around the MEA electrode tracks. Figure 3A shows a magnified image of one electrode track. Quantitative analysis on the number of neuronal cell bodies in the vicinity of the electrode tips revealed a $51 \%$ reduction in the number of neuronal cell bodies within a $100-\mu \mathrm{m}$ radius around the electrode tips of the implanted tissue compared with control tissue (Figure 3B).

\section{Wavelet Transformation}

We used the discrete wavelet transformation (DWT) method to filter the neural signal into several frequency subbands. The wavelet subbands were further classified into the SU $\left(\mathrm{W}_{0}-\mathrm{W}_{3}\right)$, MU $\left(W_{4}-W_{5}\right)$ and LFP $\left(W_{6}-W_{9}\right)$ categories as described in the Methods section. Figure 4 shows the correlation between the normalized mean wavelet power (MWP) in the wavelet bands $\mathrm{W}_{0}-\mathrm{W}_{3}$ corresponding to the $\mathrm{SU}$ frequency range and the amplitude (peak-topeak voltage $\left.\left[\mathrm{V}_{p p}\right]\right)$ of SU spikes over time. A high correlation coefficient of $0.91 \pm$ 

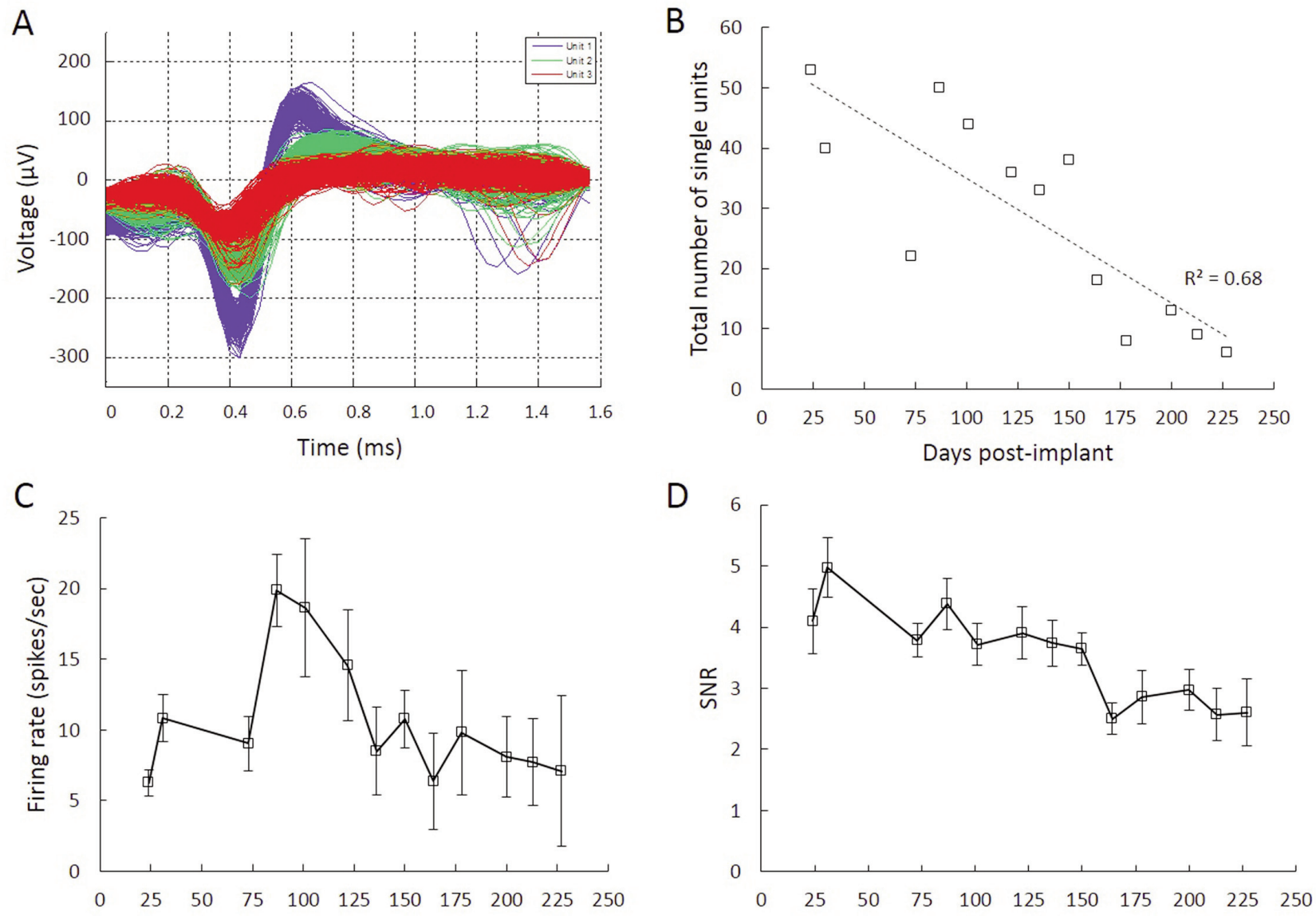

Days post-implant

Figure 2. Electrophysiology recordings. (A) Waveforms of three single units recorded from a single channel of the 96-channel MEA implanted in the primary motor cortex of the monkey. (B) A net decline in the number of single units was observed over time. Neural spikes were observed as early as $24 \mathrm{~d}$ postimplantation and were observed as late as $227 \mathrm{~d}$ postimplantation. Similar trends were observed for (C) the mean firing rate and (D) spike SNR over time. Error bars represent S.E.M.

$0.02(p<0.001)$ between the normalized MWP in the wavelet bands $W_{0}-W_{3}$ and $\mathrm{V}_{p p}$ indicated that the wavelets were efficiently tracking spikes and verified that wavelet transformation was able to efficiently decompose the raw signal.

We also compared the temporal evolution of neural signals in the SU, MU, and LFP frequency ranges. We observed a slow but significant decline over time in the MWP of wavelet bands $\mathrm{W}_{\mathrm{o}}\left(R^{2}=0.67\right.$, slope $=-0.3, p<0.001), \mathrm{W}_{1}\left(R^{2}=0.69\right.$, slope $=-0.65, p<0.001)$ and $\mathrm{W}_{2}\left(R^{2}=0.72\right.$, slope $=-1.1, p<0.001)$ and $\mathrm{W}_{3}\left(R^{2}=0.65\right.$, slope $=-1.8, p<0.05)$ corresponding to the SU frequency range (Figure $5 \mathrm{~A}$ ). Overall the MWP at d 227 in the SU frequency bands was on an average $36.4 \pm$
$1.8 \%$ lower compared with that on $\mathrm{d} 24$. In comparison we did not observe any statistically significant trends in the MWP of the wavelet bands corresponding to the MU and LFP frequency ranges indicating that the signal strength in the MU and LFP bands was stable over time (Figures 5B, C).

\section{Coherence}

From Figure 5 it was clear that the signal power in the SU bands declined over time while that in the MU and LFP frequency bands remained more stable. We next examined whether the signals in these frequency subbands were independent or correlated. To investigate this, we performed coherence analysis to calculate signal independence between all pairs of electrodes in the SU, MU and LFP frequency domains on 87 postimplantation (Figure 6). We observed that coherence decreased as the distance between electrode pairs increased and that the distancedependent decrease in coherence was more pronounced in the LFP band (slope = $\left.-0.18, R^{2}=0.96, p<0.001\right)$ compared with MU (slope $=-0.04, R^{2}=0.53, p<0.001$ ) and SU (slope $=-0.02, R^{2}=0.83, p<0.001$ ) frequency bands. More importantly, we observed that for a given interelectrode distance, the signals in the MU and SU frequency bands were significantly less coherent compared with those in the LFP frequency bands. For example, at an interelectrode distance of $1.6 \mathrm{~mm}$, the average 

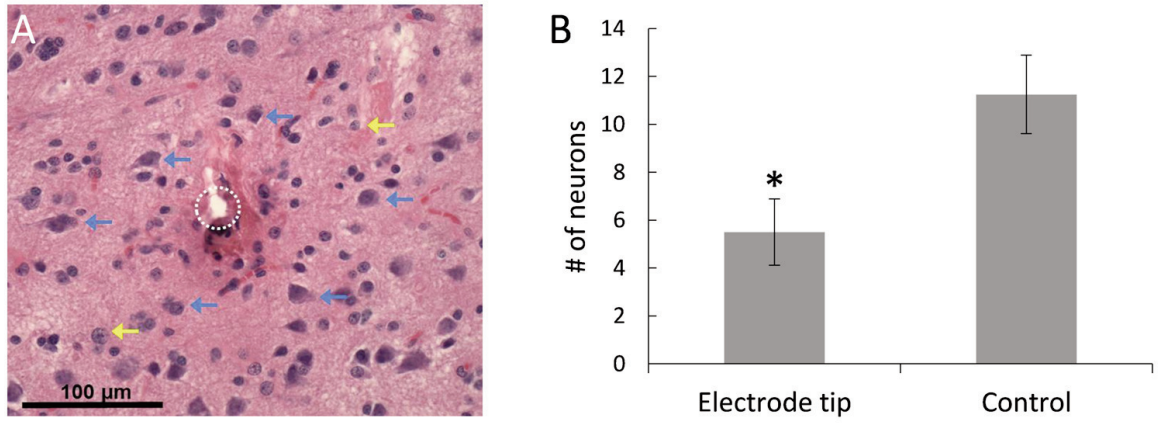

Figure 3. Histology indicates local neurodegeneration. Hematoxylin and eosin (H\&E) images of tissue section showing implant site and surrounding cells. (A) The site of electrode tip is circled. Many pyramidal neuronal cell bodies can be seen (blue arrows). Astrocytes are indicated by yellow arrows; (B) Quantitative analysis revealed a 51\% reduction in the number of neurons within a $100-\mu \mathrm{m}$ radius around the electrode tip compared with control. * $p<0.05$. Error bars represent S.E.M. coherence in MU and SU subbands was 5.5-fold $(p<0.001)$ and 8.4-fold $(p<0.001)$ lower, respectively, compared with the

There is also little information in the literature on the spatial scale at which the signals in different frequency domains become independent. Our results indicate that signals between two electrodes in the LFP frequency bands become independent (coherence $<0.3$ ) only when the separation between electrodes is at least $3.12 \mathrm{~mm}$ (coherence $=0.26 \pm$ $0.03)$. By contrast, the coherence in the MU $(0.22 \pm 0.03)$ and SU $(0.10 \pm 0.01)$ frequency bands was low even between electrodes that are next to each other (interelectrode distance $=0.4 \mathrm{~mm}$ )

\section{DISCUSSION}

In this study, we implanted a 96-channel multielectrode array into the primary motor cortex of a nonhuman primate and recorded long-term neural activity in the freely behaving animal. Our results show that: i) electrophysiological signals are detectable for more than 7.5 months after implantation; ii) the voltage amplitude and firing rate of SUs degrade over time and may be explained by neurodegeneration around electrode tips; iii) wavelet decomposition of neural signals in different frequency subbands can provide additional information on the spectral and temporal evolution of the signal; LFP subband. iv) and signals in the MU frequency subbands not only are more stable over time compared with signals in the SU frequency subbands, but also are less correlated across the array compared with signals in LFP frequency subbands.

The M1 primary motor cortex was chosen as the implantation site in our study because of the relevance of this region to motor disabilities such as spinal cord injury and stroke [for a review see (23)] as well as pain $(24,25)$, that can be potential targets for bioelectronic medicine intervention and therapy. We detected electro- physiological signals that are relevant to sensorimotor activity such as SUs, MUs and LFPs for up to 7.5 months following implantation. However, there was a significant decline in the number of SUs recorded over time. Three weeks postimplantation we recorded a peak of 53 SUs in one session while at 7.5-months postimplantation we were able to detect only 6 SUs (Figure 2B). This decline in recorded SUs was accompanied by a corresponding decline in the neuronal firing rate (Figure $2 \mathrm{C}$ ), as well as the $\mathrm{V}_{p p}$ amplitude (Figure 4). Friere et al. observed a similar decline in the number of recorded neurons and firing rate from multielectrode implants in rats over a 6-month period (4). Spike SNR was also monitored during the study. SNR can provide quantitative information on the quality of signals and electrode reliability. Similar to the firing rate and $\mathrm{V}_{p p}$, we saw variations in SNR over recording sessions with an overall statistical trend of declining SNR over time (Figure 2D). Other groups have observed similar variations in recorded signals $(6,19)$. The early variations in SNR can be attributed to the dynamic nature of tissue remodeling as well as the potential changes in neuronal excitability produced by the bioactive molecules released during the bleeding/healing

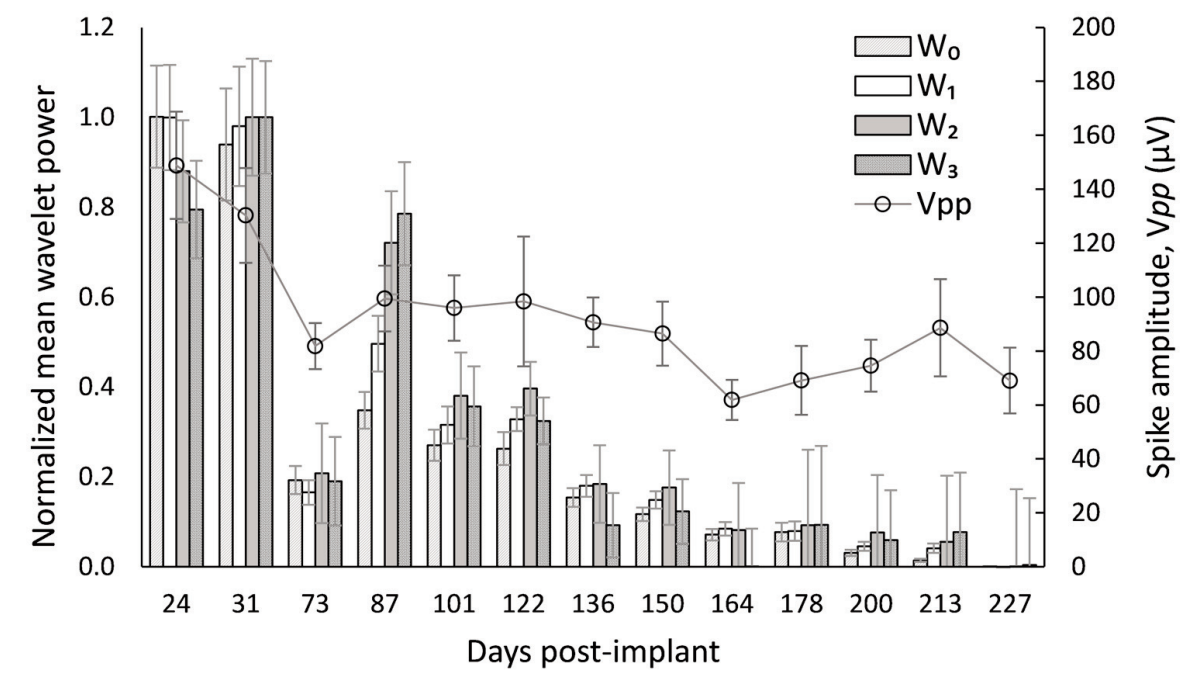

Figure 4. Wavelet power correlates with SU amplitude. A high correlation between normalized MWP and peak-to-peak voltage amplitude $\left(V_{p p}\right)$ of SU spikes was observed for the entire duration of the experiment. Error bar represents S.E.M. 
processes after the insertion trauma (19). The later variations in SNR can be attributed to a combination of neurodegeneration as well as electrode migration through cortical layers (5), which can result in different background cortical activity being recorded for each recording session (26).

We also monitored electrode impedance during the course of the study. Electrode impedance not only reveals important information regarding the electrical properties of chronic implants, including integrity of insulation coating and electrical connections, but can also give insights into the dielectric properties of the tissue-electrode interface, which can change due to protein adsorption and changes in tissue conductivity. We observed large fluctuations in the average electrode impedance during the first 2 months after implantation. This fluctuation is not unusual for recording electrode arrays and could be related to the dynamic nature of the tissue microenvironment which is in a state of constant flux postsurgery due to tissue healing processes and resulting tissue conductivity changes around the electrodes. Overall, we observed that by $75 \mathrm{~d}$ postimplantation the mean electrode impedance dropped to $273 \mathrm{k} \Omega$ and stabilized around this value for the remaining duration of the study (Supplementary Figure S1). Our results on impedance measurements are in agreement with other reports in the literature where researchers observed a similar trend in impedance of chronically implanted electrode arrays $(5,27,28)$. Long-term stable impedance can indicate that either a dynamic equilibrium has been reached at the electrode-tissue interface and/or that there was no failure of the electrode insulation. In their study, Prasad et al. (29) observed an overall increase in the electrode impedance that correlated with decreased neuronal count from the arrays. On the other hand, Kipke et al. observed a doubling of impedance over a 47-d implant period using Michigan type flat silicon electrodes implanted in rats but observed no change in recording quality (30). These

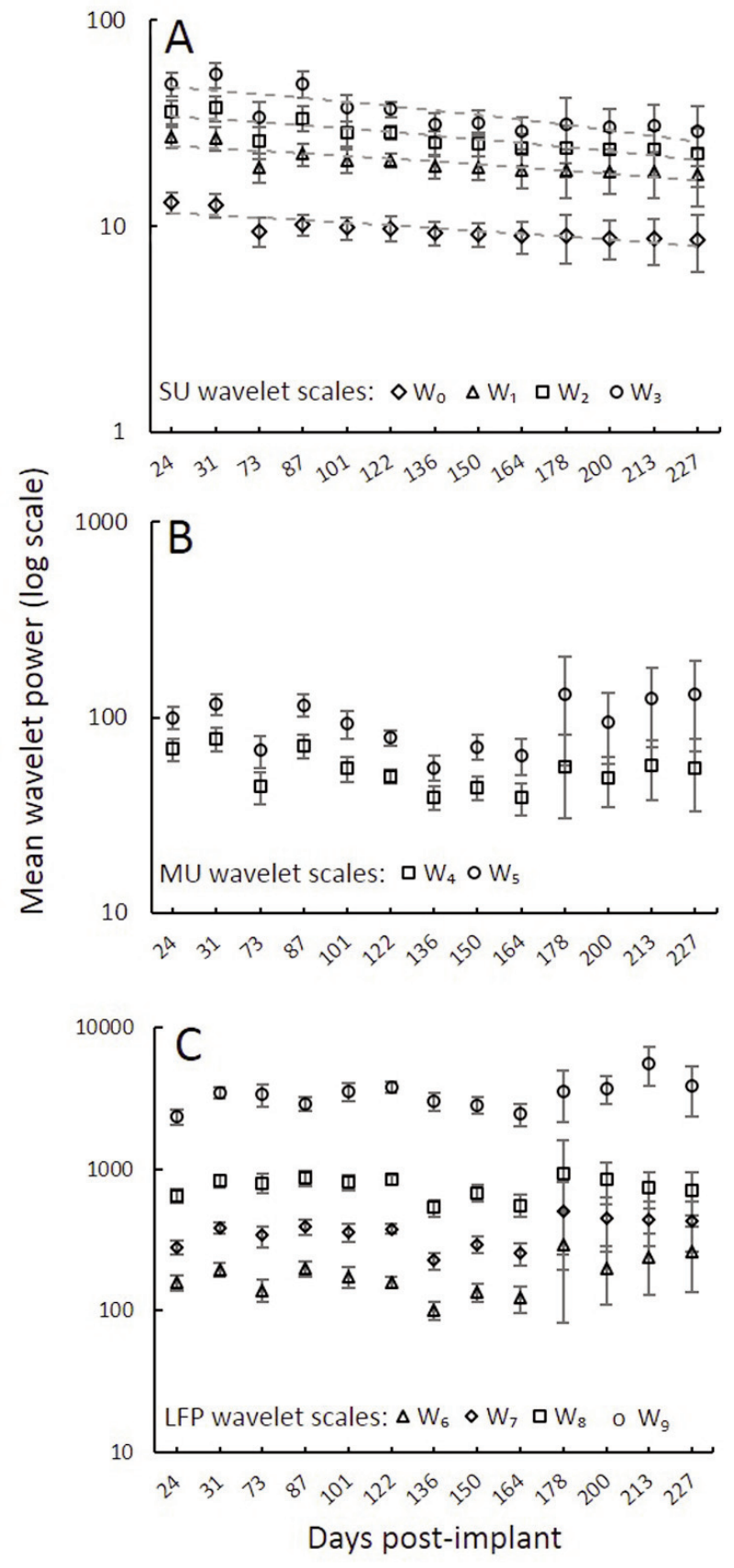

Figure 5. Temporal evolution of the MWP. (A) A steady decline in the MWP of the wavelet bands corresponding to the SU frequency range was observed. (B, C) No statistically significant trends in the MWP of the wavelet bands in the MU and LFP frequency ranges was observed. Trendlines represents a linear fit.

contrasting results further highlight that while impedance measurement can give useful information on the electrical properties of the electrode array, it is not a reliable metric to predict the ability of an electrode to record neural signals.
We also performed a basic histological examination of the explanted tissue to gain further insights into the electrode-tissue interface. We observed that the site of some of the recording tips was encapsulated by what appeared to be rims of glial 


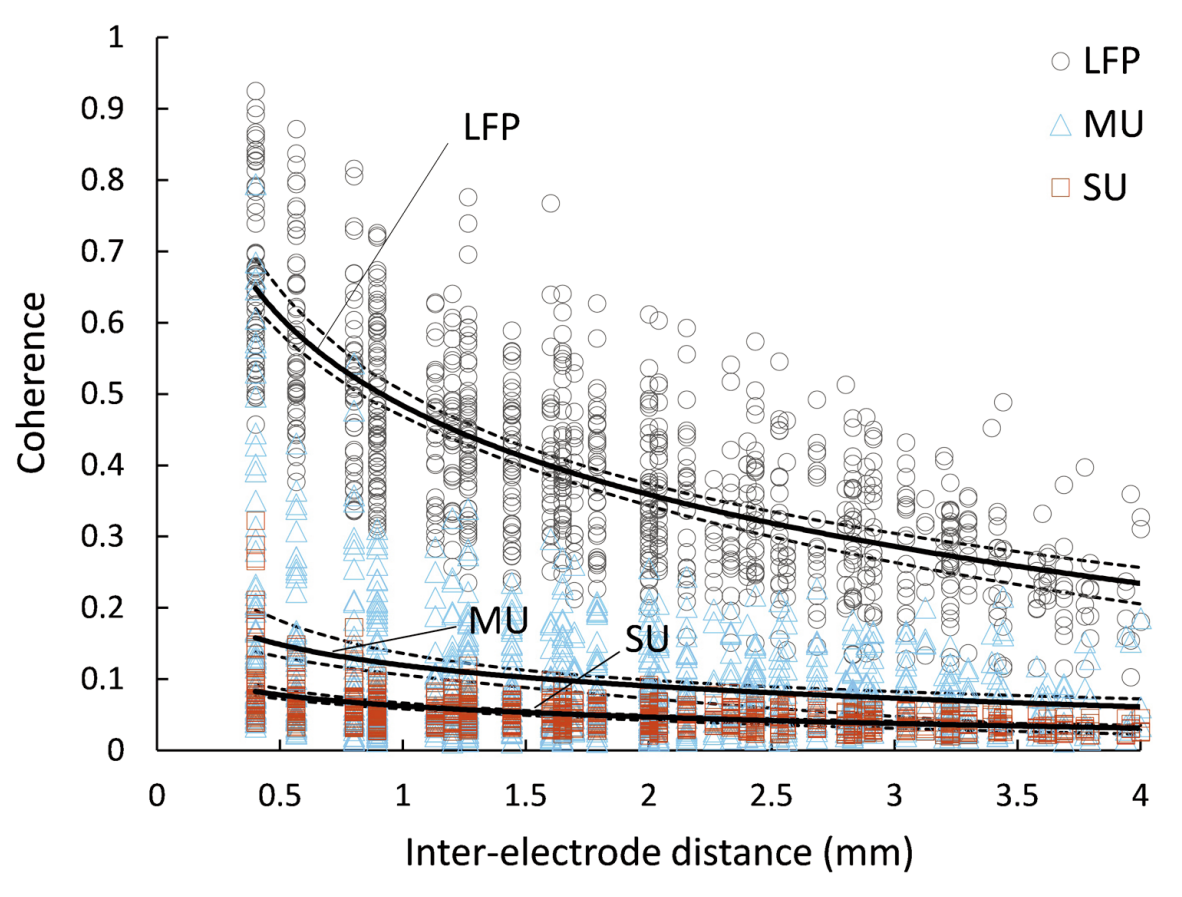

Figure 6. Coherence analysis between electrode pairs. Signal coherence on d 87 was calculated between all possible pairs of electrodes in the SU, MU and LFP frequency bands. Average coherence decreased as the distance between the electrodes increased. Coherence in the MU and SU frequency subbands was significantly lower compared with that in the LFP subbands. Solid lines represent a logarithmic fit. Broken lines represents $95 \%$ confidence bands.

fibers or scar tissue (Figure 3A). More importantly, we observed a $51 \%$ decrease in the number of neuronal cell bodies around the electrode tips compared with control tissue (Figure 3B). This data, when viewed in conjunction with the progressive loss in the number of neuronal SUs being recorded, as well as the decline in the neuronal firing rate, peak-to-peak voltage amplitude and SNR (Figures 2B-D and Figure 4), points to chronic local neurodegeneration around the electrodes. This underscores the importance of utilizing other neural signals such as MUs for neural decoding. It is now believed that local neurodegeneration around chronic electrodes is primarily initiated by microglia which migrate to the site of the electrode injury and secrete cytokines and reactive oxygen species (ROS) which have neurotoxic effects [reviewed in (31)]. We should also point out that just the presence of neuronal cell bodies alone cannot guarantee reliable recordings. The neurons must not only have a healthy cell body, but they must also maintain the normal network connectivity, including axonal and dendritic synapses, to be electrophysiologically active (9).

To gain further insight into the spectral and temporal evolution of neural signals, we performed a wavelet transformation on the raw signal. A wavelet transformation efficiently decomposes the neural signal into different frequency subbands while maintaining its temporal characteristics (32). Wavelet transformation-based analysis permits investigation of the unique features, if any, in the different frequency subbands present in the complex time-varying physiological signal. Using a wavelet transformation, we filtered the raw signal into 10 frequency subbands, as listed in the Methods section. Each of these frequency subbands can be further categorized in to $\mathrm{SU}\left(\mathrm{W}_{0}-\mathrm{W}_{3}\right), \mathrm{MU}\left(\mathrm{W}_{4}-\mathrm{W}_{5}\right)$ and LFP $\left(W_{6}-W_{9}\right)$ frequency bands. As shown in Figure 4, we observed a strong correlation between the normalized MWP in the SU wavelet bands, and the $\mathrm{V}_{p p}$ amplitude of the SU spiking activity. This confirmed the applicability of the wavelet transformation method to efficiently decompose the raw neural signal into appropriate frequency subbands.

The wavelet decomposition of each neural signal into frequency subbands also enabled us to compare the signal quality in SU, MU and LFP frequency ranges. We observed that the MWP in the SU frequency bands declined over time with the MWP on d 227, on average, being 36\% lower than that on 24 (Figure 5A). The decline in the MWP of SU frequencies is not surprising and correlates with the overall decline in the number of SUs being recording (Figure 2B). By comparison, no statistically significant trends were observed in the MWP of the MU and LFP wavelet bands over time. As mentioned earlier, MU and LFP activity is an ensemble activity of a population of neuronal processes and is less likely to degrade rapidly with local neurodegeneration around electrode tips. Therefore, it is entirely possible that, with sufficient spacing between the recording electrodes, MU activity representing neurons between the electrodes and outside the region $(>100 \mu \mathrm{m})$ of local neurodegeneration around individual electrode tips (9) could be utilized for neural decoding applications.

It is clear from the preceding discussion that SU activity degrades over time and that signals in the lower frequency MU and LFP subbands remain more stable over time. However, at lower frequencies, nearby electrodes can pick up highly correlated signals, thereby limiting the number of independent measurements that can be obtained from the electrode array (14). Indeed, we found the signals in the LFP frequency subbands to be highly correlated, even for electrode pairs that were $\sim 3 \mathrm{~mm}$ apart (Figure 6). By contrast, signals in the MU frequency subbands were not only significantly less correlated compared with those in the LFP subbands, but coherence in the MU subbands was low even between neighboring electrodes. These results suggest that MU activity can offer improved spatial resolution for neural decoding compared with LFP activity. 


\section{CONCLUSION}

Our findings on the properties of SU recording and local neurodegeneration are consistent with reports where researchers observed neural signal loss in chronic settings and neuronal death in the vicinity of implanted electrode arrays $(7,9,33)$ and highlight the drawback of relying only on SU recording for neural sensing and decoding applications. More importantly, our findings on the stability and relative signal strength of the MU signals, in conjunction with their low coherence, highlight that MU activity can be an optimal signal to use for neural decoding and analysis. Indeed, researchers are starting to develop methods to better analyze MU activity (15) and use it for neural modulation applications (14). Our study serves as the basis for guiding future strategies aimed at improving the reliability of signals recorded from implanted electrode arrays used for neuroprosthetic, as well as bioelectronic medicine applications. For example, it may prove more beneficial for chronic neural implants to decode and analyze both SU and MU activity, starting with a combined SU and MU activity during the first few months postimplantation and switching to $\mathrm{MU}$ activity thereafter. The combined recording, decoding and analysis not only can generate a better neural representation of information, but also can provide more stable signals in chronic applications such as in CNS/PNS interfaces. Future work will examine the effect of the choice of wavelet subbands on decoding efficiency and performance of chronic implants.

\section{ACKNOWLEDGMENTS}

The authors would like to thank Austin Morgan for his help with data analysis. This work was supported by independent research and development funding from Battelle Memorial Institute.

\section{DISCLOSURE}

The authors declare that they have no competing interests as defined by Bioelectronic Medicine, or other interests that might be perceived to influence the results and discussion reported in this paper.

\section{REFERENCES}

1. Bouton C. (2015) Neural decoding and applications in bioelectronic medicine. Bioelectron. Med. 2:20-4.

2. Andersen RA, Musallam S, Pesaran B. (2004) Selecting the signals for a brain-machine interface. Curr. Opin. Neurobiol. 14:720-6.

3. Bansal AK, Truccolo W, Vargas-Irwin CE, Donoghue JP. (2012) Decoding 3D reach and grasp from hybrid signals in motor and premotor cortices: spikes, multiunit activity, and local field potentials. J. Neurophysiol. 107:1337-55.

4. Freire MA, et al. (2011) Comprehensive analysis of tissue preservation and recording quality from chronic multielectrode implants. Plos One. 6:e27554.

5. Rousche PJ, Normann RA. (1998) Chronic recording capability of the Utah Intracortical Electrode Array in cat sensory cortex. J. Neurosci. Methods. 82:1-15.

6. Williams JC, Rennaker RL, Kipke DR. (1999) Long-term neural recording characteristics of wire microelectrode arrays implanted in cerebral cortex. Brain Res. Brain Res. Protoc. 4:303-13.

7. Biran R, Martin DC, Tresco PA. (2005) Neuronal cell loss accompanies the brain tissue response to chronically implanted silicon microelectrode arrays. Exp. Neurol. 195:115-26.

8. Liu X, et al. (1999) Stability of the interface between neural tissue and chronically implanted intracortical microelectrodes. IEEE Trans. Rehabil. Eng. 7:315-26.

9. McConnell GC, et al. (2009) Implanted neural electrodes cause chronic, local inflammation that is correlated with local neurodegeneration. J. Neural Eng. 6:056003.

10. Roitbak T, Sykova E. (1999) Diffusion barriers evoked in the rat cortex by reactive astrogliosis. Glia. 28:40-8.

11. Buzsaki G. (2004) Large-scale recording of neuronal ensembles. Nat. Neurosci. 7(5):446-51.

12. Scherberger H, Jarvis MR, Andersen RA. (2005) Cortical local field potential encodes movement intentions in the posterior parietal cortex. $\mathrm{Neu}$ ron. 46:347-54.

13. Scheid MR, Flint RD, Wright ZA, Slutzky MW. (2013) Long-term, stable behavior of local field potentials during brain machine interface use. Conf. Proc. IEEE Eng. Med. Biol. Soc. 2013:307-10.

14. Stark E, Abeles M. (2007) Predicting movement from multiunit activity. J. Neurosci. 27:8387-94.

15. Choi YS, Koenig MA, Jia X, Thakor NV. (2010) Quantifying time-varying multiunit neural activity using entropy based measures. IEEE Trans. Biomed. Eng. 57.

16. Quotb A, Bornat Y, Renaud S. (2011) Wavelet transform for real-time detection of action potentials in neural signals. Front. Neuroeng. 4:7.

17. Romcy-Pereira RN, de Araujo DB, Leite JP, Garcia-Cairasco N. (2008) A semi-automated algorithm for studying neuronal oscillatory patterns: a wavelet-based time frequency and coherence analysis. J. Neurosci. Methods. 167:384-92.

18. Wang Z, Maier A, Leopold DA, Logothetis NK, Liang H. (2007) Single-trial evoked potential estimation using wavelets. Comput. Biol. Med.37:463-73.
19. Suner S, Fellows MR, Vargas-Irwin C, Nakata GK, Donoghue JP. (2005) Reliability of signals from a chronically implanted, silicon-based electrode array in non-human primate primary motor cortex. IEEE Trans. Neural. Syst. Rehabil. Eng. 13:524-41.

20. Daubechies I. (1992) Ten Lectures on Wavelets. Philadelphia: Society for Industrial and Applied Mathematics. 357 pp. (CBMS-NSF Regional Conference Series in Applied Mathematics; vol. 61).

21. Farina D, do Nascimento OF, Lucas MF, Doncarli C. (2007) Optimization of wavelets for classification of movement-related cortical potentials generated by variation of force-related parameters. J. Neurosci. Methods. 162:357-63.

22. Samar VJ, Bopardikar A, Rao R, Swartz K. (1999) Wavelet analysis of neuroelectric waveforms: a conceptual tutorial. Brain Lang. 66:7-60.

23. Schwartz AB. (2004) Cortical neural prosthetics. Annu. Rev. Neurosci. 27:487-507.

24. Saitoh Y, Yoshimine T. (2007) Stimulation of primary motor cortex for intractable deafferentation pain. Acta Neurochir. Suppl. 97(Pt 2):51-6.

25. Castillo Saavedra L, Mendonca M, Fregni F. (2014) Role of the primary motor cortex in the maintenance and treatment of pain in fibromyalgia. Med. Hypotheses. 83:332-6.

26. DeYoe EA. (1983) An investigation in the awake macaque of the threshold for the detection of electrical currents applied to striate cortex: psychophysical properties and laminar differences [dissertation]. Rochester (NY): University of Rochester.

27. Chen KH, et al. (2014) The effect of chronic intracortical microstimulation on the electrode-tissue interface. J. Neural Eng. 11:026004.

28. Schmidt EM, Bak MJ, McIntosh JS. (1976) Longterm chronic recording from cortical neurons. Exp. Neurol. 52:496-506.

29. Prasad A, Sanchez JC. (2012) Quantifying long-term microelectrode array functionality using chronic in vivo impedance testing. J. Neural Eng. 9:026028.

30. Kipke DR, Vetter RJ, Williams JC, Hetke JF. (2003) Silicon-substrate intracortical microelectrode arrays for long-term recording of neuronal spike activity in cerebral cortex. IEEE Trans. Neural. Syst. Rehabil. Eng. 11:151-5.

31. Polikov VS, Tresco PA, Reichert WM. (2005) Response of brain tissue to chronically implanted neural electrodes. J. Neurosci. Methods. 148:1-18.

32. Brychta RJ, et al. (2007) Wavelet methods for spike detection in mouse renal sympathetic nerve activity. IEEE Trans. Biomed. Eng. 54:82-93.

33. Potter KA, Buck AC, Self WK, Capadona JR. (2012) Stab injury and device implantation within the brain results in inversely multiphasic neuroinflammatory and neurodegenerative responses. J. Neural Eng. 9:046020.

Cite this article as: Sharma G, et al. (2015) Time stability and coherence analysis of multiunit, singleunit and local field potential neuronal signals in chronically implanted brain electrodes. Bioelectron. Med. 2:63-71. 